\title{
Accuracy and other quality indicators of solid pancreatic mass endoscopic ultrasound-guided fine needle aspiration and biopsy in two academic endoscopy centers
}

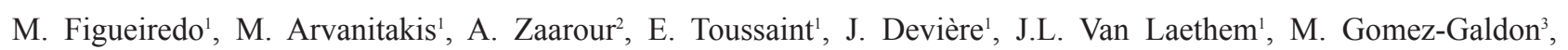
L. Verset ${ }^{3,4}$, P. Demetter, ${ }^{3,4}$, P. Eisendrath ${ }^{1,2}$

(1) Department of Gastroenterology, Hepatopancreatology and Digestive oncology, Erasme Hospital, Université Libre de Bruxelles, Belgium ; (2) Department of Gastroenterology and Hepatology, Saint-Pierre University Hospital, Université Libre de Bruxelles, Belgium ; (3) Department of Pathology, Institut Jules Bordet, Université Libre de Bruxelles, Belgium; (4) Department of Pathology, Erasme Hospital, Université Libre de Bruxelles, Bruxelles, Belgium.

\section{Abstract}

Background and aims: Endoscopic ultrasound fine-needle aspiration/biopsy (EUS-FNA/FNB) is highly accurate, but discrepancies between cytological and surgical diagnoses are still observed. We aimed to determine its accuracy and monitor quality indicators in our facilities.

Patients and methods: We performed a retrospective review of all cases of pancreatic solid lesions evaluated by EUS-FNA/FNB, between July 2015 and June 2018, in two centers. Cytological and surgical findings were categorized into five groups: benign, malignant, suspect of malignancy, undetermined and insufficient for diagnosis. Final diagnosis was based on surgical diagnosis and, in patients who did not undergo surgery, on clinical outcome after 6 months follow-up.

Results: Altogether, 142 patients were included. FNA was the preferred tissue acquisition method $(88 \%)$, with a predilection for the FNA $22 \mathrm{G}$ needle (57\%). Cytology was insufficient for diagnosis in $\mathbf{2}$ cases, therefore a full diagnostic sample was available in $\mathbf{9 8 . 6 \%}$ of the patients $(>90 \%$, ESGE target). Fifty-five $(38.7 \%)$ patients underwent surgery. In term of cancer diagnosis, comparison with final surgical pathology $(n=55)$ revealed $89 \%$ true positives, $5.5 \%$ true negatives, $3.6 \%$ false positives and $1.8 \%$ false negatives. When combining surgical diagnosis and clinical outcomes together, EUS-guided sampling sensitivity was $\mathbf{9 7 . 4 \%}$ (92.5-99.5), specificity was $92.3 \%$ (74.9-99.1), positive predictive value was $98.2 \%(93.6-$ 99.5), negative predictive value was $88.9 \%$ (72.3-96.1) and accuracy was $96.4 \%$ (91.9-98.8). Post-procedural acute pancreatitis was reported in 2 patients $(1.4 \%)$.

Conclusions: These results reveal a performance for diagnostic tissue sampling well above the ESGE proposed target standard. Also, the uncommon high specificity illustrates the determining role of the pathologist's final interpretation and diagnosis. (Acta gastroenterol. belg., 2021, 84, 451-455).

Key words: Endoscopic ultrasound-guided sampling, fine-needle aspiration/biopsy, pancreas, accuracy, quality monitoring.

\section{Introduction}

Since it was first reported in 1992 (1), endoscopic ultrasound fine-needle aspiration (EUS-FNA) has become the method of choice for the pathological diagnosis of solid pancreatic masses (fig. 1). This is not only because of its highly accurate performance regarding tissue acquisition of the target mass, with a sensitivity and specificity ranging from $85 \%$ to $92 \%$ and $96 \%$ to $98 \%$, respectively (2-5), but also because it provides an advanced staging method that allows the sampling of locoregional and distant secondary lesions that may be undetected by other imaging techniques (6). Additionally, the more recent development of endoscopic
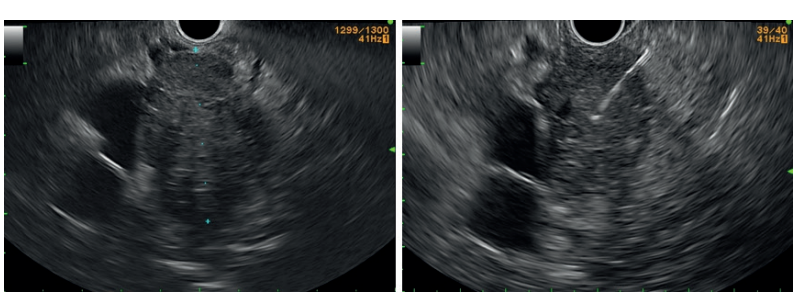

Figure 1. - Borderline resectable pancreatic ductal adenocarcinoma : tissue sample by EUS-FNB with a $22 \mathrm{G}$ forktip needle (Acquire $\AA$ ).

ultrasound fine-needle biopsy (EUS-FNB) techniques has enabled endoscopic histologic sampling allowing for molecular analysis, which is mainly useful in neoadjuvant and palliative settings. Finally, EUS-guided sampling has also proved to be a minimally invasive and safe procedure, with a very low complication rate $(3,7,8)$.

However, even though this technique has proven its benefits and is increasingly used, it still stands as one of the most sophisticated endoscopic procedures, with a long flat learning curve (9). In an effort to help clinicians and endoscopic units improve their practice, the American Society of Gastrointestinal Endoscopy (ASGE) and the European Society of Gastrointestinal Endoscopy (ESGE) have published new guidelines about technical and quality parameters concerning EUS performance $(10,11)$. Both documents suggest the characterization of the tumor features and the diagnostic value of the procedure in patients with pancreatic lesions as priority quality features. To evaluate this last parameter, ASGE has established a target diagnostic rate for malignancy of at least $70 \%$, all kind of pancreatic lesions confounded, and a sensitivity of malignancy performance target of $85 \%$. The ESGE recommendations focused on the frequency of successfully obtaining a full diagnostic tissue sample after EUS-guided sampling of a pancreatic solid lesion, which should be of at least $85 \%$ (minimum standard),

Correspondence to : Mariana Figueiredo Ferreira, MD, Department of Gastroenterology, Hepatopancreatology and Digestive Oncology, Erasme Hospital, 808 Route de Lennik, 1070 Brussels, Belgium.

E-mail : mariana.figueiredo.ferreira@erasme.ulb.ac.be

Submission date : 16/06/2020

Acceptance date : 02/03/2021 
with a target standard of $90 \%$. According to ASGE, adverse events should also be considered a major quality indicator and be below $2 \%$ for acute pancreatitis, $0.5 \%$ for perforation and $1 \%$ for clinically significant bleeding.

For the purpose of self-evaluation and self-improvement, we applied these quality criteria to all the cases of pancreatic solid lesions evaluated by EUS-guided sampling during the last three years and determined the diagnostic yield related to the aforementioned procedure in our institutions.

\section{Materials and methods}

\section{Study population}

All consecutive pancreatic solid lesions evaluated by EUS-guided sampling during a three years period (between July of 2015 and June of 2018), in the Department of Gastroenterology of two academic tertiary-care centers (Hôpital Erasme and Hôpital Saint-Pierre), in Brussels, were identified and reviewed retrospectively. The suspicion of a pancreatic solid mass lesion was based on previous radiological findings (transabdominal ultrasonography, CT scan or magnetic resonance imaging) and confirmed by EUS in all included cases.

Patients who did not undergo surgery and had a followup period shorter than 6 months were excluded, with the exception of those who deceased before, from obvious cancer related complications. The study was approved by the Ethics Committee of both hospitals (04/02/2019, ref P2019/138). Written informed consent was not necessary, given the observational and retrospective design of the study, with no inclusion of any possible patient's identifier. The study protocol conforms to the ethical guidelines of the 1975 Declaration of Helsinki as reflected in a priori approval by the institution's human research committee.

\section{Data collection}

Data collected included patient demographics (age, gender, medical and surgical history), tumor characteristics (tumor size and localization) and procedure details. Post-procedural complications as acute pancreatitis (defined accordingly to the revised Atlanta classification $(12,13))$, clinically significant bleeding (including all patients requiring blood transfusion and/or local treatment by endoscopy or arterial embolization), perforation and infection (fever and high C-reactive protein with no other apparent infectious source) were also noted.

Cytological and surgically obtained histological findings were categorized into five groups (benign, malignant, suspect of malignancy, atypical and insufficient for diagnosis), accordingly to the terminology used in the final pathology reports. Both malignant and suspect of malignancy cases were considered positive results for malignancy. All the EUS-FNA/FNB results were compared either to surgical findings alone (gold standard) or to clinical outcome, including patients deceased from obvious cancer progression within 6 months and patients followed-up for at least 6 months.

\section{EUS technique}

EUS was performed by 5 different and competent endoscopists (with more than 200 EUS examinations experience (14)), using linear EUS scope (GFUCT180, Olympus, Hamburg, Germany or EG38-70UTK, Pentax, Hamburg, Germany). All EUS were performed under deep sedation or general anesthesia. After FNA/B, samples were collected in Formaldehyde and/or Cytorich solution. In case of rapid on site examination (ROSE), performed if available, a GI pathologist analyzed the sample stained by May-Grünwald Giemsa stain in the endoscopy room. ROSE results were included in the endoscopy report. The samples were then analyzed by an experienced GI pathologist.

\section{Statistical analysis}

Statistical analyses were performed using SPSS software version 25 (IBM SPSS, NY USA). Performance characteristics as sensitivity, specificity, positive predictive value, negative predictive value and overall diagnostic accuracy were calculated. These variables are shown as percentages with $95 \%$ confidence intervals (CI 95\%). Continuous variable results were reported as medians [with minimal and maximal values reported].

\section{Results}

\section{Population characteristics}

The initial population sample included 174 patients with pancreatic solid lesions evaluated by EUSguided sampling. One patient was excluded given that radiologically suspected pancreatic lesion was not found at EUS. Thirty-three patients were excluded, due to lost to follow-up. Two of the patients underwent the procedure twice. Subsequently, data from 140 patients, corresponding to 142 EUS procedures, were analyzed (fig. 2). The majority of patients were male (54.9\%). The median age was 65 [24-89] years old. Almost twenty percent $(19.7 \%)$ of patients had chronic pancreatitis and $3.5 \%$ had their anatomy altered by a previous Whipple procedure. EUS-guided sampling had already been attempted in another hospital without success in $9.2 \%$ of the cases. Of the total cohort, only 55 patients (38.7 $\%)$ were submitted to surgery after the EUS procedure (Table 1).

\section{Tumor characteristics and technical data}

The localization of the lesions was as follows: head (45.3\%), body (35.3\%), tail (11.4\%), uncinate process 


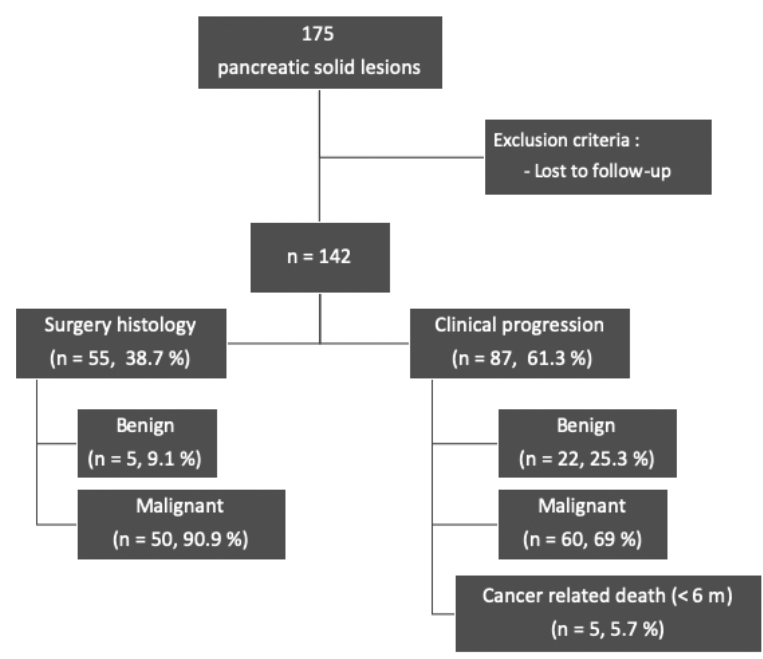

Figure 2. - Flow chart.

Table 1. - Patient and procedures characteristics $(n=142)$

\begin{tabular}{|l|c|}
\hline \multicolumn{2}{|c|}{ Patient and procedures characteristics $(\mathrm{n}=142)$} \\
\hline Male & $78(54.9 \%)$ \\
\hline Median age (years) & $65[24-89]$ \\
\hline Chronic pancreatitis & $28(19.7 \%)$ \\
\hline Previous Whipple procedure & $5(3.5 \%)$ \\
\hline Previous unsuccessful EUS-guided & $13(9.2 \%)$ \\
sampling attempt & \\
\hline Tumor localization & $64(45.3 \%)$ \\
Head & $50(35.3 \%)$ \\
Body & $16(11.4 \%)$ \\
Tail & $8(5.7 \%)$ \\
Uncinate process & $3(2.3 \%)$ \\
Not described & $26[\mathrm{IQR} 14]$ \\
\hline Tumor size median $(\mathrm{mm})$ & \\
\hline Hospital & $98(769 \%)$ \\
Erasme & $44(31 \%)$ \\
Saint-Pierre & \\
\hline Type of needle & $16(11.4 \%)$ \\
FNA 19 G & $81(57 \%)$ \\
FNA 22 G & $27(19 \%)$ \\
FNA 25 G & $9(6.3 \%)$ \\
FNB-like needle & $9(6.3 \%)$ \\
Unknown & \\
\hline ROSE & $69(48.6 \%)$ \\
Yes & $68(47.2 \%)$ \\
No & $6(4.2 \%)$ \\
Missing &
\end{tabular}

Legend : EUS $=$ endoscopic ultrasound ; FNA $=$ fine-needle aspiration ; $\mathrm{FNB}=$ fine-needle biopsy $;$ ROSE $=$ rapid on site examinatio.

$(5.7 \%)$. Location was not described in $2.3 \%$ of the cases. Only $3.5 \%$ of the lesions were smaller than $10 \mathrm{~mm}$, the smallest measuring $5 \mathrm{~mm}$ and the largest measuring $80 \mathrm{~mm}$. Size was not mentioned in $10.6 \%$ of the cases and accurate measurement was not possible because of undefined/non visible borders in $4.9 \%$.

Procedures were performed in Erasme Hospital and Saint Pierre Hospital in $69 \%$ and $31 \%$ of cases, respectively. FNA-needles (19G, $22 \mathrm{G}$ and $25 \mathrm{G}$ ) were majorly used $(87.4 \%$ ), with a preference for the FNA $22 \mathrm{G}$ needle, used in $57 \%$ of the cases. FNB-like needles were used in only $6.3 \%$ of all cases. The type of needle used was not reported in $6.3 \%$ of patients. The median number of needle passes was 3 [1-8] for the whole cohort. ROSE was only available in Erasme Hospital and was reported in $70 \%$ of cases performed in the aforementioned center, which corresponds to roughly half $(48.6 \%)$ of the whole study population.

\section{Cytological results and performance measurements}

Cytology was positive for malignancy in $79.6 \%$ of cases and considered as benign in $19 \%$. It was considered insufficient for diagnosis in 2 cases (1.4\%), leading to a full diagnostic sample available for $98.6 \%$ of the patients. There were no atypical results reported.

In term of cancer diagnosis, regarding 55 patients who underwent subsequent surgical resection, comparison with surgical pathology (gold standard) revealed 49 (89.1 $\%)$ true positives, $3(5.5 \%)$ true negatives, 2 (3.6\%) false positives and $1(1.8 \%)$ false negative. The corresponding sensitivity was $98 \%$ (95\% CI, 89.4-99.9), specificity was $60 \%$ (95\% CI, 14.7-94.7), positive predictive value was $96.1 \%$ (95\% CI, 89.3-98.7), negative predictive value was $75 \%(95 \% \mathrm{CI}, 27.5-95.9)$ and accuracy was 94.6\% (95\% CI, 84.9-98.9). The 2 patients with false positive cytology results, actually suffered from chronic pancreatitis and auto-immune pancreatitis.

By combining surgical and clinical outcomes, regarding the whole study population $(n=142)$, EUSguided sampling sensitivity for cancer was $97.4 \%(95 \%$ CI, 92.5\%-99.5\%), specificity was $92.3 \%$ (95\% CI, $74.9-99.0)$, positive predictive value was $98.2 \%(95 \%$ CI, 93.6-99.5), negative predictive value was $88.9 \%$ (95\% CI, 72.3-96.1) and accuracy was $96.4 \%$ (95\% CI, 91.9-98.8), (table 2).

Table 2. - EUS-FNA/B guided sampling diagnostic value in the two institutions

\begin{tabular}{|l|c|c|}
\hline $\begin{array}{c}\text { Diagnostic value } \\
\text { measurements } \\
(\text { CI 95\%) }\end{array}$ & $\begin{array}{c}\text { Surgical diagnosis } \\
(\mathrm{n}=55)\end{array}$ & $\begin{array}{c}\text { Surgical diagnosis } \\
\text { and clinical outcome } \\
(\mathrm{n}=142)\end{array}$ \\
\hline Sensitivity (\%) & $\begin{array}{c}98 \% \\
(89.4-99.9)\end{array}$ & $\begin{array}{c}97.3 \% \\
(92.2-99.4)\end{array}$ \\
\hline Specificity (\%) & $\begin{array}{c}60 \% \\
(14.7-94.7)\end{array}$ & $\begin{array}{c}92.3 \% \\
(74.9-99.1)\end{array}$ \\
\hline PPV (\%) & $\begin{array}{c}96.1 \% \\
(89.3-98.6)\end{array}$ & $\begin{array}{c}98.2 \% \\
(93.4-99.5)\end{array}$ \\
\hline NPV (\%) & $\begin{array}{c}75 \% \\
(27.5-95.9)\end{array}$ & $\begin{array}{c}88.9 \% \\
(72.3-96.1)\end{array}$ \\
\hline & $94.6 \%$ & $96.3 \%$ \\
& $(84.9-98.9)$ & $(91.6-98.8)$ \\
\hline
\end{tabular}

There was no significant difference between institutions, with 96.2\% (95\% CI, 90.5-98.9\%) accuracy for Erasme Hospital and $100 \%$ (95\% CI, 92.6-100\%) accuracy for Saint-Pierre Hospital.

Concerning ROSE, we observed an $91.3 \%$ agreement rate between the ROSE and the definitive laboratory cytology results, with 4 false negatives and 2 non contributive cases (against 1 false negative, 2 false positives and 2 non contributive cases for definitive cytology). In terms of accuracy, there was no significant difference 
between ROSE and non-ROSE groups, with 97.1\% (95\% CI, 89.9\%-99.7\%) and 97\% (95\% CI, 89.6\%-99.6\%), respectively. Additionally, the median number of passes was also similar in both groups (ROSE: 3 [1-8] and nonROSE: 3 [1-5]).

\section{Complications}

Post-procedural acute pancreatitis was found in 2 patients $(1,4 \%)$. No other adverse events were reported.

\section{Discussion}

The diagnostic performance values of EUS FNA/B in our two centers are encouraging and the results fulfil with the criteria proposed by the ASGE and ESGE: sensitivity for malignancy of $97.4 \%$ (ASGE performance target: $\geq$ $85 \%$ ) and frequency of obtaining full diagnostic sample of $98.6 \%$ (ESGE performance target: $>90 \%$ ).

Moreover, other performance measures were also favorable, with high values for accuracy and specificity enhancing the positive outcomes .

Even though these numbers were comparable to the results from previously published studies, we noted an inversion between sensitivity and specificity values. Indeed, in our study, sensitivity $(97.4 \%)$ was higher than specificity $(92.3 \%)$, in opposition to what was observed in a recent published metanalysis by Banafea $\mathrm{O}$ et al, which included twenty studies involving a total of 2761 patients and showed a pooled sensitivity and specificity of $90.8 \%$ and $96.5 \%$, respectively (2-5). It is clear that a significant part of the final performance of FNA/B procedures necessarily behoove to the pathologist, as during interpretation of final cyto- or histological results, pathologists may influence the sensitivity/specificity ratio depending on their confidence for considering a sample as positive for malignancy. The fact that the pathologist involved in the final interpretation of our findings has a significant experience in pancreato-biliary pathology interpretation could thus explain the observed inversion of the sensitivity/specificity ratio.

Also on the contrary of what has been suggested by some previous studies (15-17), there was no significant difference between the EUS-FNA/B accuracy of the group in which ROSE was performed against the one in which it was not. Moreover, the number of needle passes was similar in both groups too. These results could be due to the presence of ROSE procedures that were not declared on the final report so they should be interpreted with caution. Additionally, the contribution of ROSE to the EUS-FNA diagnostic accuracy has for long been a highly debated matter, with multiple studies showing discordant results and hence with the ESGE panel recommending EUS-FNA with or without ROSE equally $(18,19)$. Currently, there is an ongoing multicentric randomized non-inferiority trial (FROSENOR) with the aim of establishing if the use of the more recently developed FNB needles could undoubtedly overcome the need of ROSE for good (20). Nonetheless, considering the present available knowledge, we still find ROSE could be of value in our centers, mostly because it allows a closer collaboration between endoscopists and pathologists, contributing to their learning and to the improvement of the technique's accuracy.

Previous prospective studies have also shown conflicting results regarding the advantages of FNB over FNA-tissue sampling $(21,22)$. The most recent systematic review and metanalysis including 11 randomized control trials comparing both techniques has demonstrated no significant difference between them with regards to diagnostic yield (OR 0.61, CI $95 \% 0.28-1.33$ ) and accuracy (OR 0.85 , CI 95\% 0.53 1.36) (23). However, all of these studies concerned the first generations of FNB-type needles. New models such as the Franseen or the fork-tip needle resemble a conventional FNA needle with a modified tip design and have shown to provide high yield of histologic tissue samples and high diagnostic accuracy, as well as similar technical facility in use as FNA needles $(24,25)$. Overall, in the absence of ROSE, FNB needles require a lower number of needle passes to achieve a similar diagnostic yield compared to FNA needles, without greater technical difficulties or associated adverse effects (26). Therefore, the small number of procedures in which FNB-like needles were used in our centers $(6.3 \%)$ does not reflect the endoscopists' preferences, but instead is a direct consequence of their more recent introduction in the market and of the initial delay to obtain an authorized reimbursement by public assurance in our country.

Regarding other quality indicators, like the tumor features description, our results show a high adherence to the recommendations with low percentages of no reported tumor size or localization, though there still is place for improvement.

Finally, the low rate of post-EUS guided sampling adverse events in our centers testifies for this technique's already well-established safety (27).

This study stands out as the first EUS-FNA/B quality monitoring report that has been conceived accordingly to the recently published ESGE performance measures guidelines.

Even though it has a retrospective design, its results have allowed us to position ourselves in the "European quality scale" and, most importantly, to identify the main existing problems in our centers and, thus, be able to work on their improvement. Also, even if there was a non-neglectable number of patients lost to follow-up (probably due to the fact that our hospitals are endoscopy tertiary centers), the studied population was still vast enough to enable us to draw statistically significant results.

In conclusion, the results of this retrospective analysis reveal a performance for diagnostic tissue sampling well above the ESGE proposed target standard. Also, the uncommon high specificity illustrates the determining role of the pathologist's final interpretation and diagnosis, 
which should also be taken into account in quality assessment of endoscopic diagnostic procedures.

\section{Conflict of interests}

The authors have no conflicts of interest to declare.

\section{References}

1. VILMANN P, JACOBSEN GK, HENRIKSEN FW, HANCKE S. Endoscopic ultrasonography with guided fine needle aspiration biopsy in pancreatic disease. Gastrointest. Endosc. $1992 \mathrm{Apr}, 38(2)$ : 172-3.

2. PULI SR, KALVAN, BECHTOLD ML, PAMULAPARTHY SR, CASHMAN MD, ESTES NC, et al. Diagnostic accuracy of endoscopic ultrasound in pancreatic neuroendocrine tumors : a systematic review and meta analysis. World J. Gastroenterol. 2013 Jun 21, 19(23) : 3678-84.

3. CHEN J, YANG R, LU Y, XIA Y, ZHOU H. Diagnostic accuracy of endoscopic ultrasound-guided fine-needle aspiration for solid pancreatic lesion : a systematic review. J. Cancer Res. Clin. Oncol. 2012 Sep, 138(9) : 1433-41.

4. BANAFEA O, MGHANGA FP, ZHAO J, ZHAO R, ZHU L. Endoscopic ultrasonography with fine-needle aspiration for histological diagnosis of solid pancreatic masses: a meta-analysis of diagnostic accuracy studies. $B M C$ Gastroenterol. 2016 Aug 31, 16 : 108.

5. GRESS F, GOTTLIEB K, SHERMAN S, LEHMAN G. Endoscopic ultrasonography-guided fine-needle aspiration biopsy of suspected pancreatic cancer. Ann. Intern. Med. 2001 Mar 20, 134(6) : 459-64.

6. SUZUKI R, IRISAWA A, BHUTANI MS, HIKICHI T, TAKAGI T, SHIBUKAWA G, et al. An automated spring-loaded needle for endoscopic ultrasound-guided abdominal paracentesis in cancer patients. World $J$ Gastrointest. Endosc. 2014 Feb 16, 6(2) : 55-9.

7. ELOUBEIDI MA, TAMHANE A, VARADARAJULU S, WILCOX CM. Frequency of major complications after EUS-guided FNA of solid pancreatic masses: a prospective evaluation. Gastrointest. Endosc. 2006 Apr, 63(4) $622-9$

8. BANG JY, HEBERT-MAGEE S, TREVINO J, RAMESH J, VARADARAJULU S. Randomized trial comparing the 22-gauge aspiration and 22-gauge biopsy needles for EUS-guided sampling of solid pancreatic mass lesions. Gastrointest. Endosc. 2012 Aug, 76(2) : 321-7.

9. RÖSCH T. State of the art lecture: Endoscopic ultrasonography: Training and competence. Endoscopy. 2006 Jun, 38(S 1) : 69-72.

10. DOMAGK D, OPPONG KW, AABAKKEN L, CZAKÓ L, GYÖKERES T, MANES G, et al. Performance measures for ERCP and endoscopic ultrasound : a European Society of Gastrointestinal Endoscopy (ESGE) Quality Improvement Initiative. Endoscopy. 2018, 50(11) : 1116-27.

11. WANI S, WALLACE MB, COHEN J, PIKE IM, ADLER DG, KOCHMAN ML, et al. Quality indicators for EUS. Gastrointest. Endosc. 2015 Jan, 81(1) : 67-80.

12. BANKS PA, BOLLEN TL, DERVENIS C, GOOSZEN HG, JOHNSON CD, SARR MG, et al. Classification of acute pancreatitis, 2012 : revision of the Atlanta classification and definitions by international consensus. Gut. 2013 Jan, 62(1) : 102-11.

13. LANKISCH PG, APTE M, BANKS PA. Acute pancreatitis. The Lancet. 2015 Jul, 386(9988) : 85-96.
14. WANI S, HAN S, SIMON V, HALL M, EARLY D, AAGAARD E, et al. Setting minimum standards for training in EUS and ERCP : results from a prospective multicenter study evaluating learning curves and competence among advanced endoscopy trainees. Gastrointest. Endosc. 2019 Jun, 89(6) : 1160-1168.e9.

15. IGLESIAS-GARCIA J, DOMINGUEZ-MUNOZ JE, ABDULKADER I, LARINO-NOIA J, EUGENYEVA E, LOZANO-LEON A, et al. Influence of on-site cytopathology evaluation on the diagnostic accuracy of endoscopic ultrasound-guided fine needle aspiration (EUS-FNA) of solid pancreatic masses. Am. J. Gastroenterol. 2011 Sep, 106(9) : 1705-10.

16. THOMAS T, KAYE PV, RAGUNATH K, AITHAL G. Efficacy, safety, and predictive factors for a positive yield of EUS-guided Trucut biopsy : a large tertiary referral center experience. Am. J. Gastroenterol. 2009 Mar, 104(3) : 584-91.

17. KLAPMAN JB, LOGRONO R, DYE CE, WAXMAN I. Clinical impact of on-site cytopathology interpretation on endoscopic ultrasound-guided fine needle aspiration. Am. J. Gastroenterol. 2003 Jun, 98(6) : 1289-94.

18. YANG F, LIU E, SUN S. Rapid on-site evaluation (ROSE) with EUS-FNA : The ROSE Slooks beautiful. Endosc. Ultrasound. 2019, 8(5) : 283.

19. POLKOWSKI M, JENSSEN C, KAYE P, CARRARA S, DEPREZ P, GINES A, et al. Technical aspects of endoscopic ultrasound (EUS)-guided sampling in gastroenterology : European Society of Gastrointestinal Endoscopy (ESGE) Technical Guideline - March 2017. Endoscopy. 2017 Oct, 49(10) : 989-1006.

20. CRINÒ SF, MANFRIN E, SCARPA A, BALDAQUE-SILVA F, CARRARA $\mathrm{S}$, DE NUCCI G, et al. EUS-FNB with or without on-site evaluation for the diagnosis of solid pancreatic lesions (FROSENOR) : Protocol for a multicenter randomized non-inferiority trial. Dig. Liver Dis. 2019 Jun, 51(6) : 901-6.

21. CHENG B, ZHANG Y, CHEN Q, SUN B, DENG Z, SHAN H, et al. Analysis of Fine-Needle Biopsy vs Fine-Needle Aspiration in Diagnosis of Pancreatic and Abdominal Masses : A Prospective, Multicenter, Randomized Controlled Trial. Clin. Gastroenterol. Hepatol. 2018 Aug, 16(8) : 1314-21.

22. NAGULA S, POURMAND K, ASLANIAN H, BUCOBO JC, GONDA TA, GONZALEZ S, et al. Comparison of Endoscopic Ultrasound-Fine-Needle Aspiration and Endoscopic Ultrasound-Fine-Needle Biopsy for Solid Lesions in a Multicenter, Randomized Trial. Clin. Gastroenterol. Hepatol. 2018 Aug, 16(8) : 1307-1313.e1.

23. MACHICADO JD, THOSANI N, WANI S. Will Abandoning Fine-Needle Aspiration Increase Diagnostic Yield From Tissues Collected During Endoscopic Ultrasound? Clin. Gastroenterol. Hepatol. 2018 Aug, 16(8) : 1203-6.

24. WITT BL, ADLER DG, HILDEN K, LAYFIELD LJ. A comparative needle study : EUS-FNA procedures using the HD ProCore ${ }^{\mathrm{TM}}$ and EchoTip ${ }^{\text {}}$ 22-gauge needle types : Comparative Needle Study. Diagn. Cytopathol. 2013 Dec, 41(12) : 1069-74.

25. BANG JY, HEBERT-MAGEE S, NAVANEETHAN U, HASAN MK, HAWES R, VARADARAJULU S. Randomized trial comparing the Franseen and Fork-tip needles for EUS-guided fine-needle biopsy sampling of solid pancreatic mass lesions. Gastrointest. Endosc. 2018 Jun, 87(6) : 1432-8.

26. KHAN M, GRIMM I, ALI B, NOLLAN R, TOMBAZZI C, ISMAIL M, et al. A meta-analysis of endoscopic ultrasound-fine-needle aspiration compared to endoscopic ultrasound-fine-needle biopsy : diagnostic yield and the value of onsite cytopathological assessment. Endosc. Int. Open. 2017 May, 5 : E36375.

27. WANG K-X, BEN Q-W, JIN Z-D, DU Y-Q, ZOU D-W, LIAO Z, et al. Assessment of morbidity and mortality associated with EUS-guided FNA : a systematic review. Gastrointest. Endosc. $2011 \mathrm{Feb}, 73(2)$ : 283-90. 